\title{
Entanglement and Decoherence of a Micromechanical Resonator via Coupling to a Cooper-Pair Box
}

\author{
A. D. Armour, ${ }^{1}$ M. P. Blencowe, ${ }^{2}$ and K. C. Schwab ${ }^{3}$ \\ ${ }^{1}$ Blackett Laboratory, Imperial College of Science, Technology and Medicine, London SW7 2BW, United Kingdom \\ ${ }^{2}$ Department of Physics and Astronomy, Dartmouth College, Hanover, New Hampshire 03755 \\ ${ }^{3}$ Laboratory for Physical Sciences, College Park, Maryland 20740
}

(Received 12 January 2002; published 20 March 2002)

\begin{abstract}
We analyze the quantum dynamics of a micromechanical resonator capacitively coupled to a Cooperpair box. With appropriate quantum state control of the Cooper box, the resonator can be driven into a superposition of spatially separated states. The Cooper box can also be used to probe the decay of the resonator superposition state due to environmental decoherence.
\end{abstract}

DOI: $10.1103 /$ PhysRevLett.88.148301

PACS numbers: $85.85 .+\mathrm{j}, 03.65 . \mathrm{Yz}$

Micromechanical resonators with fundamental vibrational mode frequencies in the range $10 \mathrm{MHz}-1 \mathrm{GHz}$ can now be fabricated [1,2]. Applications include fast, ultrasensitive force and displacement detectors [3], electrometers [4,5], and radio frequency signal processors [6]. Advances in the development of micromechanical devices also raise the fundamental question of whether mechanical systems containing macroscopic numbers of atoms will exhibit quantum behavior. Because of their size, quantum behavior in micromechanical systems will be strongly influenced by interactions with the environment and the existence of an experimentally accessible quantum regime will depend on the rate at which decoherence occurs [7].

In this Letter, we analyze an experimentally implementable scheme to create and detect superpositions of macroscopically distinct quantum states in a micromechanical resonator, and furthermore measure their decoherence rates, by entangling the resonator with a Cooper box [8-10]. The key advantage over optomechanical schemes $[7,11]$ is the demonstrated coherent control of the Cooper box quantum charge state [9], together with the strong (controllable) coupling which can be achieved between the Cooper box state and the motional degree of freedom of a micron-sized mechanical oscillator. Cooper box-based schemes have also been proposed for creating macroscopic quantum state superpositions in superconducting islands [12] and superconducting resonators [13].

A Cooper box consists of a small superconducting island weakly linked to a superconducting reservoir [8-10]. The state of the Cooper box is determined by the balance between its Coulomb charging energy, and the strength of the Cooper-pair tunneling between the island and reservoir. Using an external gate, the Cooper box can be driven into either of two states of definite Cooper-pair number or a linear superposition of the two states [9]. Cooper boxes are being explored as possible candidates for qubits in future quantum computing devices since they act as readily controllable two-level quantum systems $[10,14]$.

The electrostatic interaction between a conducting cantilever and a nearby Cooper box causes a displacement in the cantilever whose sign depends on which of the two charge states the Cooper box is in. When the Cooper box is prepared in a superposition of charge states, it and the cantilever become entangled and the cantilever is driven into a superposition of spatially separated states. If the coupling is strong enough, then the separation between the states in the superposition can become larger than their quantum position uncertainty, and so we can describe them as macroscopically distinct. Again using external voltage gates, the degree of entanglement between the cantilever and the Cooper box after a given period of interaction (which we call the wait time) can be imprinted on the charge state of the box. For an isolated cantilever the entanglement between the cantilever and the Cooper box is a periodic function of the wait time. However, because the cantilever is driven into a superposition of spatially separated states it will be subject to environmental decoherence which eventually destroys the periodicity in the entanglement between the cantilever and the Cooper box. Of course the Cooper box itself is also subject to environmental decoherence, but this should not prevent the decoherence rate of the cantilever being determined (as we discuss below).

The charge state of the Cooper box can be measured with great sensitivity and with minimum disturbance using a radio-frequency single electron transistor (rf-SET) [15]. Probing the charge state of the box after different wait times, and averaged over many different runs, will give information about the periodicity in the degree of entanglement of the cantilever and the Cooper box. Furthermore, measurement of the charge state of the Cooper box after different wait times will also allow the decoherence time of the cantilever due to interactions with its environment to be inferred. The circuit diagram for the system is shown in Fig. 1.

Let us first focus on the dynamics of the Cooper boxcoupled cantilever system, neglecting the coupling to the cantilever environment and the rf-SET. The Hamiltonian is

$H=4 E_{\mathrm{C}} \delta n \hat{\sigma}_{z}-\frac{1}{2} E_{\mathrm{J}} \hat{\sigma}_{x}+\hbar \omega_{\mathrm{m}} \hat{a}^{\dagger} \hat{a}+\lambda\left(\hat{a}+\hat{a}^{\dagger}\right) \hat{\sigma}_{z}$, 


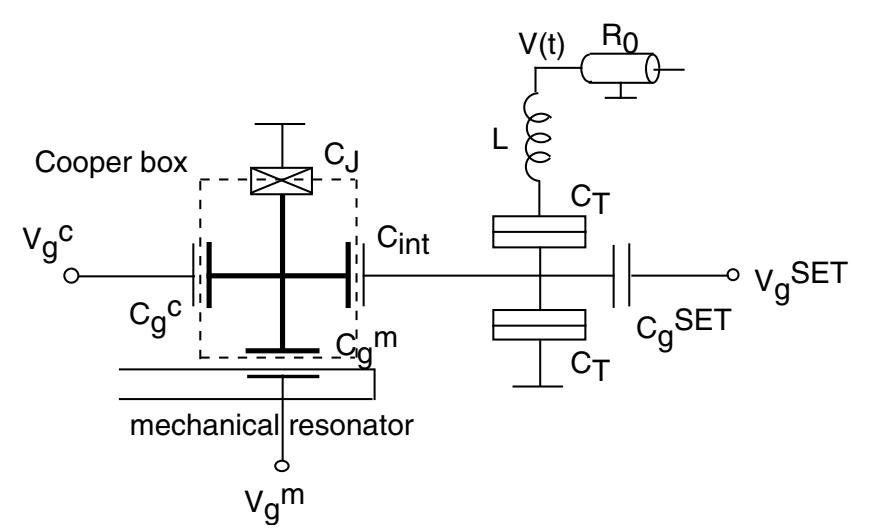

FIG. 1. Circuit diagram for the coupled Cooper box-cantilever system and the rf-SET.

where $\delta n=n_{\mathrm{g}}-(n+1 / 2)$ with $n_{\mathrm{g}}=-\left(C_{\mathrm{g}}^{\mathrm{c}} V_{\mathrm{g}}^{\mathrm{c}}+\right.$ $\left.C_{\mathrm{g}}^{\mathrm{m}} V_{\mathrm{g}}^{\mathrm{m}}\right) / 2 e$ the dimensionless, total gate charge. The control gate voltage $V_{\mathrm{g}}^{\mathrm{c}}$ and cantilever gate electrode voltage $V_{\mathrm{g}}^{\mathrm{m}}$ ranges are restricted such that $-1 / 2 \leq \delta n \leq 1 / 2$ for some chosen $n$, so that only Cooper charge states $|n\rangle \equiv|-\rangle \equiv\left(\begin{array}{l}1 \\ 0\end{array}\right)$ and $|n+1\rangle \equiv|+\rangle \equiv\left(\begin{array}{l}0 \\ 1\end{array}\right)$ play a role. Thus it is natural to use spin notation where $\hat{\sigma}_{x}$ and $\hat{\sigma}_{z}$ are the usual Pauli matrices. The coupling constant between the box and cantilever electrode is $\lambda=-4 E_{\mathrm{C}} n_{\mathrm{g}}^{\mathrm{m}} \frac{\Delta x_{\mathrm{zp}}}{d}$, where $n_{\mathrm{g}}^{\mathrm{m}}=-C_{\mathrm{g}}^{\mathrm{m}} V_{\mathrm{g}}^{\mathrm{m}} / 2 e, \Delta x_{\mathrm{zp}}$ is the zero-point displacement uncertainty of the cantilever, and $d \gg \Delta x_{\mathrm{zp}}$ is the cantilever electrode-island gap. Only the in-plane fundamental flexural mode of the cantilever, with frequency $\omega_{\mathrm{m}}$ and operators $a$ and $a^{\dagger}$, is taken into account. All other modes have a much weaker coupling to the box and will be neglected [16]. We assume that the Josephson junction capacitance $C_{\mathrm{J}} \gg C_{\mathrm{g}}^{\mathrm{c}}$ and $C_{\mathrm{g}}^{\mathrm{m}}$, so that the charging energy of the box $E_{\mathrm{C}} \approx e^{2} / 2 C_{\mathrm{J}}$.

The scheme for the control pulse sequence is indicated in Fig. 2. It is convenient to determine the evolution of the box-cantilever system using the coherent state basis for the cantilever. At $t=0$, we take as initial state $\left|\Psi_{0}\right\rangle=|-\rangle|\alpha\rangle$, where $|\alpha\rangle$ denotes a coherent state [17]. The first pulse takes the box to the degeneracy point and is of duration $T_{\mathrm{R}} / 4$, where $T_{\mathrm{R}}=h / E_{\mathrm{J}}$ is the coherent oscillation (Rabi) period of the Cooper state. The state $\left|\Psi_{0}\right\rangle$ evolves to $\left|\Psi_{T_{\mathrm{R}} / 4}\right\rangle=\frac{1}{\sqrt{2}}(|-\rangle+i|+\rangle)|\alpha\rangle$, where it is assumed that $\omega_{\mathrm{m}} T_{\mathrm{R}} \ll 1$ and the cantilever box coupling strength is such that the coherent state evolution can be neglected. Following the first pulse, there is a wait time $\tau$

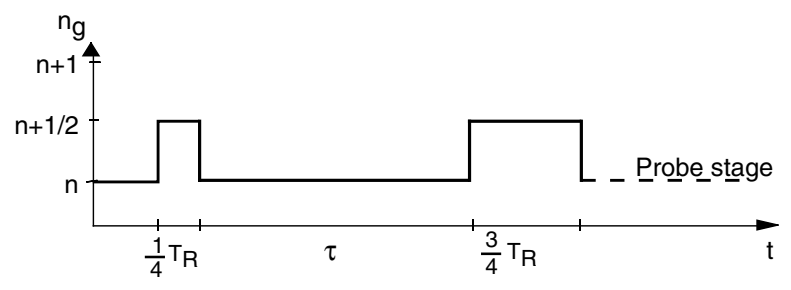

FIG. 2. Pulse sequence for manipulating the state of the box. during which the box and cantilever systems interact, resulting in an entangled state:

$$
\begin{aligned}
\left|\Psi_{T_{\mathrm{R}} / 4+\tau}\right\rangle= & \frac{1}{\sqrt{2}} e^{2 i E_{\mathrm{C}} \tau / \hbar}|-\rangle\left|\alpha_{-}(\tau)\right\rangle \\
& +\frac{i}{\sqrt{2}} e^{-2 i E_{\mathrm{C}} \tau / \hbar}|+\rangle\left|\alpha_{+}(\tau)\right\rangle,
\end{aligned}
$$

where we assume that $E_{\mathrm{J}} \ll E_{\mathrm{C}}$, and so neglect the Josephson tunneling term in the evolution, and where $\left|\alpha_{ \pm}(\tau)\right\rangle=e^{ \pm i \phi(\alpha, \tau)}\left|\alpha e^{-i \omega_{\mathrm{m}} \tau} \mp \kappa\left(1-e^{-i \omega_{\mathrm{m}} \tau}\right)\right\rangle, \quad$ with the phase $\phi(\alpha, \tau)=\frac{i \kappa}{2}\left[\alpha\left(1-e^{-i \omega_{\mathrm{m}} \tau}\right)-\alpha^{*}(1-\right.$ $\left.\left.e^{i \omega_{\mathrm{m}} \tau}\right)\right]$ and the dimensionless coupling $\kappa=\lambda / \hbar \omega_{\mathrm{m}}$. (Note we have neglected an overall, $\alpha$-independent phase term as it will not affect the final probabilities.) The spatial separation between the cantilever states $\left|\alpha_{ \pm}(\tau)\right\rangle$ is $2 \kappa\left(1-\cos \omega_{\mathrm{m}} \tau\right) \Delta x_{\mathrm{zp}}$ and, thus, the condition for the maximum separation of the states to exceed their width is $4|\kappa|>1$.

By taking the box to the degeneracy point a second time with a pulse of duration $3 T_{R} / 4$, a signature of the separated cantilever states is imprinted on the Cooper pair number probabilities:

$$
\begin{aligned}
\left|\Psi_{T_{\mathrm{R}}+\tau}\right\rangle= & \frac{1}{2}|-\rangle\left[e^{2 i E_{\mathrm{C}} \tau / \hbar}\left|\alpha_{-}(\tau)\right\rangle+e^{-2 i E_{\mathrm{C}} \tau / \hbar}\left|\alpha_{+}(\tau)\right\rangle\right] \\
& +\frac{i}{2}|+\rangle\left[e^{2 i E_{\mathrm{C}} \tau / \hbar}\left|\alpha_{-}(\tau)\right\rangle-e^{-2 i E_{\mathrm{C}} \tau / \hbar}\left|\alpha_{+}(\tau)\right\rangle\right]
\end{aligned}
$$

and

$$
\begin{gathered}
\mathrm{P}(|-\rangle)=\frac{1}{2}\left\{1+\cos \left[4 E_{\mathrm{C}} \tau / \hbar+4 \phi(\alpha, \tau)\right]\right. \\
\left.\times e^{-4 \kappa^{2}\left(1-\cos \omega_{\mathrm{m}} \tau\right)}\right\}
\end{gathered}
$$

If there is no coupling between the Cooper box and cantilever (i.e., $\kappa=0$ ), the second control pulse simply returns the box to its initial state $|-\rangle$ (the Cooper state has effectively performed a full Rabi oscillation at the degeneracy point) provided $\tau=2 \pi k \hbar / 4 E_{\mathrm{C}}, k=0,1,2 \ldots$

Assuming that, before the control pulse sequence is applied, the box-cantilever system is in a thermal equilibrium state (because $4 E_{\mathrm{C}} \gg k_{\mathrm{B}} T$, the box will be in its ground state $|-\rangle$ to a good approximation), we must thermally average the above probability. This gives

$$
\begin{aligned}
\mathrm{P}_{\mathrm{th}}(|-\rangle)=\frac{1}{2}\{1 & +\cos \left[4 E_{\mathrm{C}} \tau / \hbar+4 \kappa^{2} \sin \omega_{\mathrm{m}} \tau\right] \\
& \left.\times e^{-4 \kappa^{2}\left(1-\cos \omega_{\mathrm{m}} \tau\right)(1+2 \bar{N})}\right\},
\end{aligned}
$$

where $\bar{N}=\left(e^{\hbar \omega_{\mathrm{m}} / k_{\mathrm{B}} T}-1\right)^{-1}$ is the thermal occupation of the cantilever mode. The cosine function leads to rapid oscillations whose magnitude is controlled by the exponential term. It is convenient to define the envelope of $\mathrm{P}_{\mathrm{th}}(|-\rangle)$ as the function in Eq. (2) with the argument in the square brackets set to zero.

Notice that the envelope of Eq. (2) recovers its initial value (i.e., unity) as $\tau$ approaches the period $\tau_{\mathrm{m}}$ of the cantilever mode. This is a consequence of the harmonic nature 
of the cantilever as a measuring device for the Cooper box state; the correlations set up between the box and cantilever states are completely undone and the two systems are no longer entangled after an integral number of harmonic oscillation periods. This "recoherence" effect is discussed in Ref. [7] for a system involving a cavity field coupled to a movable mirror. Similar effects are also discussed in Refs. [12,13].

The conditions for the quantum state control are as follows:

$$
\tau_{\mathrm{j}}<\frac{h}{4 E_{\mathrm{C}}} \ll \frac{h}{E_{\mathrm{J}}} \ll \tau_{\mathrm{m}}<\tau_{\mathrm{d}}^{\mathrm{cb}},
$$

where $\tau_{\mathrm{j}}$ denotes the jitter time of the pulse sequence generator and $\tau_{\mathrm{d}}^{\mathrm{cb}}$ denotes the decoherence time of the Cooper box superposition states through processes other than due to the cantilever and its environment. The first inequality in the chain is necessary to resolve the rapid oscillations with period $h / 4 E_{\mathrm{C}}$ in Eq. (2), and thereby measure the associated envelope function; without being able to position the pulses with sufficient temporal accuracy, the oscillations would be washed out giving a constant $\mathrm{P}_{\mathrm{th}}(|-\rangle) \approx$ $1 / 2$. The last inequality is necessary to observe the recoherences and the effects of the cantilever's environment (which we discuss below). The middle two inequalities are not essential, their purpose being only to simplify the theoretical analysis and hence the description of the quantum dynamics. A 1 ps jitter time is achievable. Choosing $E_{\mathrm{C}}=150 \mu \mathrm{eV}$ gives $h / 4 E_{\mathrm{C}} \approx 7 \mathrm{ps}$, and choosing $E_{\mathrm{J}}=4 \mu \mathrm{eV}$ gives $h / E_{\mathrm{J}} \approx 1 \mathrm{~ns}$. A fundamental flexural frequency $\nu_{\mathrm{m}}=50 \mathrm{MHz}$, giving a period $\tau_{\mathrm{m}}=20 \mathrm{~ns}$, is readily achievable with micron-sized cantilevers [1,2].

The most serious practical constraint arises from the decoherence of the Cooper box itself, which if it occurs too fast will obscure the quantum dynamics of the cantilever. At present, decoherence times of only a few ns have been achieved and an improvement of about an order of magnitude would be required to implement our scheme. However, recent work by Nakamura et al. [18] has demonstrated that decoherence times of the box can be extended by applying refocusing pulses. There is no fundamental reason why the Cooper box decoherence time should be limited to less than $20 \mathrm{~ns}$ and so considerable further improvements are to be expected.

In order that the Cooper-pair superposition state separate the cantilever coherent states by more than their width (the quantum position uncertainty), we require that the coupling strength satisfies $4|\lambda| / \hbar \omega_{\mathrm{m}}>1$. A Si cantilever with dimensions $l$ (length) $\times w$ (width) $\times$ $t$ (thickness) $=1.6 \mu \mathrm{m} \times 0.1 \mu \mathrm{m} \times 0.1 \mu \mathrm{m}$ has a fundamental flexural frequency $\nu_{\mathrm{m}} \approx 50 \mathrm{MHz}$ and zero-point uncertainty $\Delta x_{\mathrm{zp}} \approx 1.4 \times 10^{-3} \AA$. Assuming a cantilever electrode-Cooper island gap $d=0.1 \mu \mathrm{m}$ and gate capacitance $C_{\mathrm{g}}^{\mathrm{m}} \approx 20 \mathrm{aF}$, the dimensionless gate charge $n_{\mathrm{g}}^{\mathrm{m}} \approx-63 V_{\mathrm{g}}^{\mathrm{m}}$. Substituting in these parameter values and $E_{\mathrm{C}}=150 \mu \mathrm{eV}$, we have for the separation condition $V_{\mathrm{g}}^{\mathrm{m}}>1.0 \mathrm{~V}$. Such a voltage can be applied across a $0.1 \mu \mathrm{m}$ gap: it will deflect the cantilever by a much smaller distance than the gap and is well below the breakdown voltage.

We now turn to consider the effect of the cantilever's environment on the coupled Cooper box-cantilever dynamics. We model the environment of the cantilever as a bath of oscillators at a fixed temperature, $T$, each of which are weakly coupled to the fundamental flexural mode. This model is widely used for open systems and is equivalent to treating the cantilever mode as a damped quantum oscillator [16,17], characterized by an energy damping rate parameter, $2 \gamma \ll \omega_{\mathrm{m}}, k_{\mathrm{B}} T / \hbar$ [19]. When the calculation of $\mathrm{P}_{\mathrm{th}}(|-\rangle)$ is repeated including the coupling of the cantilever to the bath oscillators we find

$$
\mathrm{P}_{\mathrm{th}}(|-\rangle)=\frac{1}{2}\left\{1+\cos \left[4 E_{\mathrm{C}} \tau / \hbar+4 \kappa^{2} \varphi(\tau)\right] e^{-\Gamma(\tau)}\right\},
$$

where $\varphi(\tau)$ is a slowly varying phase factor which depends on the properties of the cantilever. The damping of the coherent oscillations is given by

$$
\begin{aligned}
\Gamma(\tau)=\frac{4 \lambda^{2}(2 \bar{N}+1)}{\hbar^{2}\left(\omega_{\mathrm{m}}^{2}+\gamma^{2}\right)}\{ & \gamma \tau-\frac{2 \gamma \omega_{\mathrm{m}}}{\gamma^{2}+\omega_{\mathrm{m}}^{2}} e^{-\gamma \tau} \sin \left(\omega_{\mathrm{m}} \tau\right) \\
& +\left(\frac{\gamma^{2}-\omega_{\mathrm{m}}^{2}}{\gamma^{2}+\omega_{\mathrm{m}}^{2}}\right) \\
& \left.\times\left[e^{-\gamma \tau} \cos \left(\omega_{\mathrm{m}} \tau\right)-1\right]\right\} .
\end{aligned}
$$

Again we define the envelope of $\mathrm{P}_{\mathrm{th}}(|-\rangle)$ by setting the total phase in the square brackets of Eq. (3) to zero.

The energy damping rate in the model, $2 \gamma$, can be estimated empirically by measuring the quality factor of the cantilever, $Q$, since $2 \gamma=\omega_{\mathrm{m}} / Q$. Figure 3 shows the envelope of $\mathrm{P}_{\mathrm{th}}(|-\rangle)$ when the coupling of the cantilever to

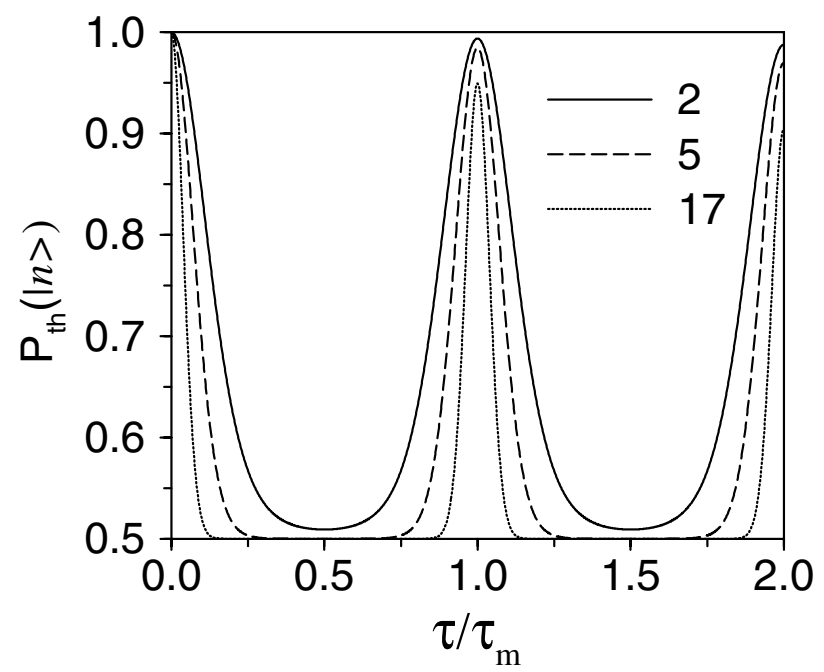

FIG. 3. Envelope of $\mathrm{P}_{\mathrm{th}}(|-\rangle)$, including the cantilever's environment, as a function of wait time for $Q=1000$. The figures in the legend correspond to the values of the quantity $4 \kappa^{2}(2 \bar{N}+1)$. 
the environment is included, for $Q=1000$ as a function of the quantity $\left(2 \lambda / \hbar \omega_{\mathrm{m}}\right)^{2}(2 \bar{N}+1)$. The series of curves shown could be obtained, for example, by setting the temperature at $30 \mathrm{mK}$ and sweeping the coupling strength $\kappa$ from 0.14 to 0.41 . In the presence of a finite damping rate, the recoherences are indeed suppressed progressively as either the temperature or the cantilever-Cooper box coupling is increased. Notice that because of the predicted dependence of the decoherence rate of the cantilever on the coupling and temperature, it would be possible to separate out the effect of the cantilever's environment from other contributions causing decoherence of the Cooper box.

The final stage in the process is to read out the charge state of the Cooper box using the rf-SET. At the end of the control stage, the rf-SET is tuned away from the Coulomb blockade region and a nonzero drain-source voltage applied, resulting in a tunneling current through the SET. As a result of the capacitive coupling $C_{\text {int }}$ between the Cooper box and SET, the SET island voltage will be affected by the Cooper box island charge. Hence, the SET tunneling current probes the Cooper box charge state. If the lifetime of the Cooper box state is determined by the rf-SET island voltage and quantum electromagnetic mode fluctuations acting back on the box, then the condition for the measurement time to be shorter than this lifetime is $[15,10,20]$

$$
\frac{\tau_{\text {decay }}}{\tau_{\text {measure }}}=\left(\frac{4 E_{\mathrm{C}}}{E_{\mathrm{J}}}\right)^{2} \frac{\hbar^{2}}{S_{\mathrm{V}}(\delta q)^{2}}>1,
$$

where $\delta q$ is the charge sensitivity of the rf-SET and $S_{\mathrm{V}}$ is the sum of the SET island and electromagnetic mode voltage noise evaluated at the Cooper state oscillation frequency $\omega=4 E_{\mathrm{C}} / \hbar$. Using the values $E_{\mathrm{C}}=150 \mu \mathrm{eV}$ and $E_{\mathrm{J}}=4 \mu \mathrm{eV}$, resulting from the above state control condition, the electromagnetic-mode dominated voltage noise $S_{\mathrm{V}}=0.14 \mathrm{nV}^{2} / \mathrm{Hz}$ at $4 E_{\mathrm{C}} / h=145 \mathrm{GHz}$, and the value for the rf-SET charge sensitivity $\delta q=6.3 \mu e / \sqrt{\mathrm{Hz}}$ determined experimentally in Ref. [15], we have $\tau_{\text {decay }} / \tau_{\text {measure }}=1.7 \times 10^{3}$. Choosing, for example, $C_{\mathrm{int}} / C_{\mathrm{J}}=0.1$, the respective times are in fact $\tau_{\text {measure }}=4 \mathrm{~ns}$ and $\tau_{\text {decay }}=7 \mu \mathrm{s}$. However, the actual lifetime is likely to be somewhat smaller than $7 \mu \mathrm{s}$, limited by Cooper box offset charge noise [18,21], but it certainly exceeds 4 ns [9]. Thus, provided $C_{\text {int }}$ is not too small, it should be possible to read out the charge state.

The scheme we have detailed provides a feasible way of probing the quantum coherence of a micromechanical system. The calculation of the decoherence rate of the cantilever due to interactions with an oscillator bath predicts that mechanical systems of the kind now available could display quantum coherence over time scales of a few periods. The analysis detailed here is readily adapted to include more elaborate pulse sequences, such as that used by Nakamura et al. [18] to increase the intrinsic decoherence time of the Cooper box, without significantly affecting the conclusions.

We thank R. Lifshitz and A. MacKinnon for useful discussions. This work was supported in part by the National Security Agency (NSA), the Advanced Research and Development Activity (ARDA), the Army Research Office (ARO), and the Engineering and Physical Sciences Research Council (EPSRC).

[1] A. N. Cleland and M. L. Roukes, Appl. Phys. Lett. 69, 2653 (1996).

[2] D. W. Carr, S. Evoy, L. Sekaric, H. G. Craighead, and J. M. Parpia, Appl. Phys. Lett. 75, 920 (1999).

[3] M.P. Blencowe and M.N. Wybourne, Appl. Phys. Lett. 77, 3845 (2000); Y. Zhang and M. P. Blencowe, cond-mat/0109412.

[4] A. N. Cleland and M. L. Roukes, Nature (London) 392, 160 (1998).

[5] A. Erbe, C. Weiss, W. Zwerger, and R. H. Blick, Phys. Rev. Lett. 87, 096106 (2001).

[6] K. Wang, A.-C. Wong, and C. T.-C. Nguyen, J. Microelectromech. Syst. 9, 347 (2000).

[7] S. Bose, K. Jacobs, and P. L. Knight, Phys. Rev. A 59, 3204 (1999).

[8] V. Bouchiat, D. Vion, P. Joyez, D. Esteve, and M. H. Devoret, Phys. Scr. T76, 165 (1998).

[9] Y. Nakamura, Yu. A. Pashkin, and J. S. Tsai, Nature (London) 398, 786 (1999).

[10] Y. Makhlin, G. Schön, and A. Shnirman, Rev. Mod. Phys. 73, 357 (2001).

[11] V. Giovannetti, S. Mancini, and P. Tombesi, quant-ph/0108044.

[12] F. Marquardt and C. Bruder, Phys. Rev. B 63, 054514 (2001).

[13] O. Buisson and F. W. J. Hekking, in Macroscopic Quantum Coherence and Quantum Computing, edited by D. V. Averin, B. Ruggiero, and P. Silvestrini (Kluwer, New York, 2001).

[14] P. Echternach, C. P. Williams, S. C. Dultz, S. Braunstein, and J. P. Dowling, Quantum Inf. Comput. 1, 143 (2001).

[15] A. Aassime, G. Johansson, G. Wendin, R. J. Schoelkopf, and P. Delsing, Phys. Rev. Lett. 86, 3376 (2001).

[16] A.D. Armour and M. P. Blencowe, Phys. Rev. B 64, 035311 (2001).

[17] L. Mandel and E. Wolf, Optical Coherence and Quantum Optics (Cambridge University Press, Cambridge, United Kingdom, 1995).

[18] Y. Nakamura, Yu. A. Pashkin, T. Yamamoto, and J. S. Tsai, cond-mat/0111402.

[19] F. Haake and R. Reibold, Phys. Rev. A 32, 2462 (1985).

[20] M. H. Devoret and R. J. Schoelkopf, Nature (London) 406, 1039 (2000).

[21] A. Cottet, A. Steinbach, P. Joyez, D. Vion, H. Pothier, D. Esteve, and M. E. Huber, in Macroscopic Quantum Coherence and Quantum Computing, edited by D. V. Averin, B. Ruggiero, and P. Silvestrini (Kluwer, New York, 2001). 